Bull. Korean Math. Soc. 52 (2015), No. 1, pp. 335-344

http://dx.doi.org/10.4134/BKMS.2015.52.1.335

\title{
NOTES ON REAL HYPERSURFACES IN A COMPLEX SPACE FORM
}

\author{
Jong TAEK Cho
}

AbSTRACT. We characterize a homogeneous real hypersurface of type (A) or a ruled real hypersurface in a non-flat complex space form, respectively.

\section{Introduction}

Let $\left(\widetilde{M}_{n}(c), J, \widetilde{g}\right)$ be an $n$-dimensional complex space form with Kählerian structure $(J, \widetilde{g})$ of constant holomorphic sectional curvature $c$ and let $M$ be an orientable real hypersurface in $\widetilde{M}_{n}(c)$. Then $M$ has an almost contact metric structure $(\eta, \phi, \xi, g)$ induced from $(J, \widetilde{g})$ (see Section 1). U.-H. Ki and Y. J. Suh [13] proved that are no real hypersurfaces in a non-flat complex space form satisfying $\phi A+A \phi=0$. From this we see that there are no almost cosymplectic or almost Kenmotsu real hypersurfaces in a non-flat complex space form (see Proposition 4 in Section 3). We put $P=\phi A+A \phi$. Then we prove that $P$ is invariant along the Reeb flow, that is, $£_{\xi} P=0$ if and only if $M$ is locally congruent to a homogeneous hypersurface of type (A) in $P_{n} \mathbb{C}$ or $H_{n} \mathbb{C}$ (Theorem $9)$.

In Section 4 , we prove that for a real hypersurface $M$ in a non-flat complex space form $\widetilde{M}_{n}(c)(c \neq 0) \phi$ is a transversal Killing tensor field, that is, $\left(\nabla_{X} \phi\right) X=0$ for any vector field $X \perp \xi$ if and only if $M$ is locally congruent to a ruled real hypersurface (Theorem 13). Also, we prove that for a real hypersurface $M$ in a non-flat complex space form $\widetilde{M}_{n}(c)(c \neq 0), n \geq 3$, the shape operator $A$ is transversally Killing (that is, $\left(\nabla_{X} A\right) X=0$ for any vector field $X \perp \xi$ ) if and only if $M$ is locally congruent to a real hypersurface of type (A) (Theorem 16).

\section{Almost contact geometry}

In this paper, all manifolds are assumed to be connected and of class $C^{\infty}$ and the real hypersurfaces are supposed to be oriented.

Received February 18, 2014; Revised May 28, 2014.

2010 Mathematics Subject Classification. 53B25, 53C15, 53C25.

Key words and phrases. real hypersurface, complex space form, almost contact structure.

This paper was financially supported by Chonnam National University, 2013. 
First, we give a brief review of several fundamental notions and formulas which we will need later on. An odd-dimensional differentiable manifold $M$ has an almost contact structure if it admits a (1,1)-tensor field $\phi$, a vector field $\xi$ and a 1-form $\eta$ satisfying

$$
\phi^{2}=-I+\eta \otimes \xi, \eta(\xi)=1 .
$$

We call $\xi$ the Reeb vector field. Then we can always find a compatible Riemannian metric $g$, namely it satisfies

$$
g(\phi X, \phi Y)=g(X, Y)-\eta(X) \eta(Y)
$$

for any vector fields $X, Y$ on $M$. We call $(\eta, \phi, \xi, g)$ an almost contact metric structure of $M$ and $M=(M, \eta, \phi, \xi, g)$ an almost contact metric manifold. From (1) and (2) we easily get

$$
\phi \xi=0, \quad \eta \circ \phi=0, \quad \eta(X)=g(X, \xi) .
$$

The tangent space $T_{p} M$ of $M$ at each point $p \in M$ is decomposed as $T_{p} M=$ $D_{p} \oplus \operatorname{Span}\{\xi\}_{p}($ direct sum $)$, where we denote $D_{p}=\left\{v \in T_{p} M \mid \eta(v)=0\right\}$. Then $D: p \rightarrow D_{p}$ defines a distribution orthogonal to $\xi$. For an almost contact metric manifold $M$, one may define naturally an almost complex structure on the product manifold $M \times \mathbb{R}$, where $\mathbb{R}$ denotes the real line. If the almost complex structure is integrable, $M$ is said to be normal. The integrability condition for the almost complex structure is the vanishing of the tensor $[\phi, \phi]+2 d \eta \otimes \xi$, where $[\phi, \phi]$ denotes the Nijenhuis torsion of $\phi$. For an almost contact metric manifold $M$, we define its fundamental 2 -form $\Phi$ by $\Phi(X, Y)=g(\phi X, Y)$. If $M$ satisfies in addition

$$
\Phi=d \eta
$$

$M$ is called a contact metric manifold. A normal contact metric manifold is called a Sasakian manifold. An almost contact metric manifold is said to be a quasi-Sasakian manifold if it is normal and $d \Phi=0$. Then we easily see that Sasakian manifolds are quasi-Sasakian. Other than a class of contact manifolds, we have mainly two classes of almost contact manifolds, that is, almost cosymplectic manifolds and almost Kenmotsu manifolds. An almost contact metric manifold $(M, \eta, \phi, \xi, g)$ is said to be almost cosymplectic if $d \eta=0$ and $d \Phi=0$. Such a class was introduced by S. I. Goldberg and K. Yano [10]. The trivial products of an almost Kählerian manifold and a real line or a circle are the simplest examples of such manifolds. An almost contact metric manifold $M$ is said to be almost Kenmotsu if $d \eta=0$ and $d \Phi=2 \eta \wedge \Phi$. The warped products of an almost Kählerian manifold and a real line give examples of almost Kenmotsu manifolds. For further properties and examples of almost Kenmotsu manifolds, we refer to [9] and [12].

For more details about the general theory of almost contact metric manifolds, we refer to [4]. 


\section{Real hypersurfaces in a complex space form}

Let $\widetilde{M}=\widetilde{M}_{n}(c)$ be a complex space form of constant holomorphic sectional curvature $c, M$ be a real hypersurface of $\widetilde{M}$ and $N$ be a unit normal vector field of $M$ in $\widetilde{M}$. We denote by $\tilde{g}$ and $J$ a Kählerian metric tensor and its complex structure tensor on $\widetilde{M}$, respectively. For any vector field $X$ tangent to $M$, we put

$$
J X=\phi X+\eta(X) N, \quad J N=-\xi
$$

where $\phi X$ is the tangential part of $J X, \phi$ a $(1,1)$-type tensor field, $\eta$ is a 1-form, and $\xi$ is a unit vector field on $M$. The induced Riemannian metric on $M$ is denoted by $g$. Then by properties of $(J, \widetilde{g})$ we see that the structure $(\eta, \phi, \xi, g)$ is an almost contact metric structure on $M$. Indeed, we can deduce (1) and (2) from (3).

The Gauss and Weingarten formula for $M$ are given as

$$
\begin{aligned}
& \widetilde{\nabla}_{X} Y=\nabla_{X} Y+g(A X, Y) N, \\
& \widetilde{\nabla}_{X} N=-A X
\end{aligned}
$$

for any tangent vector fields $X, Y$, where $\widetilde{\nabla}$ and $\nabla$ denote the Levi-Civita connections of $\left(M_{n}(c), \widetilde{g}\right)$ and $(M, g)$, respectively, and $A$ is the shape operator field. An eigenvalue and an eigenvector of the shape operator $A$ is called a principal curvature and a principal curvature vector, respectively. From (3) and $\widetilde{\nabla} J=0$, we then obtain

$$
\begin{gathered}
\left(\nabla_{X} \phi\right) Y=\eta(Y) A X-g(A X, Y) \xi \\
\nabla_{X} \xi=\phi A X .
\end{gathered}
$$

We easily see that $d \Phi(X, Y, Z)=0$ is equivalent to $\mathfrak{S}_{X, Y, Z} g\left(\left(\nabla_{X} \phi\right) Y, Z\right)=0$, where $\mathfrak{S}_{X, Y, Z}$ denotes the cyclic sum for $X, Y, Z$. Then we find from (4)

Proposition 1. Every real hypersurface in a Kählerian manifold satisfies $d \Phi=$ 0 .

Due to [13] we know that there are no real hypersurfaces in a non-flat complex space form satisfying $\phi A+A \phi=0$. Using (5) we have also:

Proposition 2. There is no real hypersurface in a non-flat complex space form whose almost contact metric structure is almost cosymplectic or almost Kenmotsu.

We have the following Gauss and Codazzi equations:

$$
\begin{aligned}
R(X, Y) Z= & \frac{c}{4}\{g(Y, Z) X-g(X, Z) Y \\
& +g(\phi Y, Z) \phi X-g(\phi X, Z) \phi Y-2 g(\phi X, Y) \phi Z\} \\
& +g(A Y, Z) A X-g(A X, Z) A Y,
\end{aligned}
$$




$$
\left(\nabla_{X} A\right) Y-\left(\nabla_{Y} A\right) X=\frac{c}{4}\{\eta(X) \phi Y-\eta(Y) \phi X-2 g(\phi X, Y) \xi\}
$$

for any tangent vector fields $X, Y, Z$ on $M$.

The following facts are needed later to prove our results.

Lemma 3 ([13], [17], [19]). If $\xi$ is a principal curvature vector, then the associated principal curvature $\alpha=g(A \xi, \xi)$ is constant.

Suppose that $M$ is a Hopf hypersurface, that is, $\xi$ is a principal curvature vector field $A \xi=\alpha \xi$. Differentiating this covariantly, then by using Lemma 3 and (5) we have

$$
\left(\nabla_{X} A\right) \xi=\alpha \phi A X-A \phi A X
$$

and further by using (7) we obtain

$$
\left(\nabla_{\xi} A\right) X=\frac{c}{4} \phi X+\alpha \phi A X-A \phi A X
$$

for any vector field $X$ on $M$. Since $\nabla_{\xi} A$ is self-adjoint, we have

$$
2 A \phi A X-\frac{c}{2} \phi X=\alpha(\phi A+A \phi) X \text {. }
$$

If we assume that $A X=\lambda X(\|X\|=1)$ for $X$ orthogonal to $\xi$, then we get

$$
(2 \lambda-\alpha) A \phi X=\left(\lambda \alpha+\frac{c}{2}\right) \phi X \text {. }
$$

We may also refer to [22, Lemma 2.2 and Corollary 2.3]. Then, we have:

Lemma 4. For a Hopf hypersurface $M$ in a non-flat complex space form $\widetilde{M}_{n}(c), \phi X$ is a principal direction if $X(\perp \xi)$ is a principal direction.

R. Takagi [28], [29] classified the homogeneous real hypersurfaces of $P_{n} \mathbb{C}$ into six types. T. E. Cecil and P. J. Ryan [6] extensively studied a Hopf hypersurface (whose Reeb vector $\xi$ is a principal curvature vector), which is realized as tubes over certain submanifolds in $P_{n} \mathbb{C}$, by using its focal map. By making use of those results, M. Kimura [15] proved the local classification theorem for Hopf hypersurfaces of $P_{n} \mathbb{C}$ whose all principal curvatures are constant.

Theorem 5 ([15]). Let $M$ be a Hopf hypersurface of $P_{n} \mathbb{C}$. Then $M$ has constant principal curvatures if and only if $M$ is locally congruent to one of the following:

$\left(\mathrm{A}_{1}\right)$ a geodesic hypersphere of radius $r$, where $0<r<\frac{\pi}{2}$,

$\left(\mathrm{A}_{2}\right)$ a tube of radius $r$ over a totally geodesic $P_{l} \mathbb{C}(1 \leq l \leq n-2)$, where $0<r<\frac{\pi}{2}$

(B) a tube of radius $r$ over a complex quadric $Q^{n-1}$ and $P_{n} \mathbb{R}$, where $0<$ $r<\frac{\pi}{4}$

(C) a tube of radius $r$ over $P_{1} \mathbb{C} \times P_{\frac{n-1}{2}} \mathbb{C}$, where $0<r<\frac{\pi}{4}$ and $n(\geq 5)$ is odd,

(D) a tube of radius $r$ over a complex Grassmann $G_{2,5} \mathbb{C}$, where $0<r<\frac{\pi}{4}$ and $n=9$, 
(E) a tube of radius $r$ over a Hermitian symmetric space $S O(10) / U(5)$, where $0<r<\frac{\pi}{4}$ and $n=15$.

For the case $H_{n} \mathbb{C}$, J. Berndt [3] proved the classification theorem for Hopf hypersurfaces whose all principal curvatures are constant.

Theorem 6 ([3]). Let $M$ be a Hopf hypersurface of $H_{n} \mathbb{C}$. Then $M$ has constant principal curvatures if and only if $M$ is locally congruent to one of the following:

$\left(\mathrm{A}_{0}\right)$ a horosphere,

$\left(\mathrm{A}_{1}\right)$ a geodesic hypersphere or a tube over a complex hyperbolic hyperplane $H_{n-1} \mathbb{C}$

$\left(\mathrm{A}_{2}\right)$ a tube over a totally geodesic $H_{l} \mathbb{C}(1 \leq l \leq n-2)$,

(B) a tube over a totally real hyperbolic space $H_{n} \mathbb{R}$.

We call simply type $(\mathrm{A})$ for real hypersurfaces of type $\left(\mathrm{A}_{1}\right),\left(\mathrm{A}_{2}\right)$ in $P_{n} \mathbb{C}$ and ones of type $\left(\mathrm{A}_{0}\right),\left(\mathrm{A}_{1}\right)$ or $\left(\mathrm{A}_{2}\right)$ in $H_{n} \mathbb{C}$.

Real hypersurfaces with $A \phi+\phi A=k \phi$ are classified by T. Adachi, M. Kameda and S. Maeda (see also Lemma 3.1 in [27] for the case $c<0$ and $n>2)$ :

Proposition 7 ([1]). Let $M$ be a real hypersurface of $\widetilde{M}_{n}(c)$ with $n \geq 2$ and $c \neq 0$. Then $M$ satisfies $\phi A+A \phi=k \phi$ for some nonzero constant $k$ if and only if $M$ is of type $\left(\mathrm{A}_{0}\right),\left(\mathrm{A}_{1}\right)$ or $(\mathrm{B})$.

Homogeneous real hypersurfaces of type (A) are characterized as follows:

Proposition 8 ([11], [20], [21], [23], [24], [25]). Let $M$ be a real hypersurface in a non-flat complex space form $\widetilde{M}_{n}(c)(n \geq 2)$. Then the following conditions are mutually equivalent:

- $M$ satisfies $A \phi=\phi A$;

- $M$ is locally congruent to a type (A) hypersurface;

- $\xi$ is a Killing vector field;

- the almost contact metric structure is normal.

In these cases, $M$ is a quasi-Sasakian manifold.

From Propositions 7 and 8, type $\left(\mathrm{A}_{2}\right)$ hypersurfaces are characterized as the only non-Sasakian quasi-Sasakian hypersurfaces in $P_{n} \mathbb{C}$ and $H_{n} \mathbb{C}$ (cf. [7], [26]).

Theorem 9. Let $M$ be a real hypersurface in a non-flat complex space form $\widetilde{M}_{n}(c)(c \neq 0)$. Then $P=\phi A+A \phi$ is invariant along the Reeb flow, that is, $£_{\xi} P=0$ if and only if $M$ is locally congruent to a homogeneous hypersurface of type $(\mathrm{A})$ in $P_{n} \mathbb{C}$ or $H_{n} \mathbb{C}$.

Proof. Let $M$ be real hypersurface in a non-flat complex space form $\widetilde{M}_{n}(c)$. Suppose that $M$ satisfies $£_{\xi} P=0$. We compute first $\left(£_{\xi} P\right) X=£_{\xi}(P X)-$ $P\left(£_{\xi} X\right)=[\xi, P X]-P[\xi, X]$, where $[\cdot, \cdot]$ denotes the Lie bracket. Then using (5) we have

$$
\left(\nabla_{\xi} P\right) X=\phi A P X-P \phi A X
$$


Develop (10) to

$$
\left(\nabla_{\xi} \phi\right) A X+\phi\left(\nabla_{\xi} A\right) X+\left(\nabla_{\xi} A\right) \phi X+A\left(\nabla_{\xi} \phi\right) X=\phi A P X-P \phi A X
$$

for any vector field $X$ on $M$. Use (4) to obtain

$$
\left(\phi\left(\nabla_{\xi} A\right)+\left(\nabla_{\xi} A\right) \phi\right) X=\eta\left(A^{2} X\right) \xi-\eta(X) A^{2} \xi+\phi A P X-P \phi A X .
$$

Since $\phi\left(\nabla_{\xi} A\right)+\left(\nabla_{\xi} A\right) \phi$ is skew-symmetric, from (12) we have

$$
\phi A P X-P \phi A X+P A \phi X-A \phi P X=0 .
$$

If we put $X=\xi$ in (13), then we get

$$
A \phi U=0,
$$

where we have put $\phi A \xi=U$. Taking the inner product with $\xi$ in (14), then it follows that $\|U\|^{2}=0$. That is, $A \xi=\alpha \xi$. Assume that $A X=\lambda X(\|X\|=1)$ for $X$ orthogonal to $\xi$, Then using (8) and (9) equation (12) yields that

$$
\lambda= \pm\left(\frac{\alpha \lambda+c / 2}{2 \lambda-\alpha}\right)
$$

when $2 \lambda \neq \alpha$. But, we know that there is no real hypersurface satisfying $\phi A+A \phi=0$ in $P_{n} \mathbb{C}$ or $H_{n} \mathbb{C}$. So, $M$ should satisfy $\lambda=(\alpha \lambda+c / 2) /(2 \lambda-\alpha)$, that is, $\phi A=A \phi$. Thus, by Proposition 8 we see that $M$ is locally congruent to type $(\mathrm{A})$ hypersurface. The remaining case $2 \lambda=\alpha$ determines a horosphere in $H_{n} \mathbb{C}$ (cf. [3]). After all, $M$ is locally congruent to a homogeneous real hypersurface of type (A).

\section{Transversal Killing tensors}

M. Kimura [16] constructed ruled real hypersurfaces, which are foliated real hypersurfaces with totally geodesic submanifolds of $P_{n} \mathbb{C}$ as leaves of codimension 1. Let $\bar{M}$ be a hypersurface in $S^{2 n+1}$ defined by

$$
\begin{gathered}
\left\{\left(r e^{i t} \cos \theta, r e^{i t} \sin \theta,\left(1-r^{2}\right)^{1 / 2} z_{2}, \ldots,\left(1-r^{2}\right)^{1 / 2} z_{n}\right) \in \mathbb{C}^{n+1} \mid\right. \\
\left.\sum_{j=2}^{n}\left|z_{j}\right|^{2}=1,0<r<1,0 \leq t, \theta<2 \pi\right\} .
\end{gathered}
$$

Then the Hopf image $M$ of $\bar{M}$ is a minimal ruled hypersurface in $P_{n} \mathbb{C}$. Actually, the shape operator is given as follows: $A \xi=r^{-1}\left(1-r^{2}\right)^{1 / 2} U, A U=r^{-1}(1-$ $\left.r^{2}\right)^{1 / 2} \xi$ and $A Z=0$ for $Z \perp \xi, U$. We note that the above example of a ruled real hypersurface is not complete. In a similar way, S.-S. Ahn, S.-B. Lee and Y. J. Suh [2] gave a minimal complete ruled real hypersurfaces in $H_{n} \mathbb{C}$. Furthermore, they are characterized by the following.

Proposition 10 ([2], [16]). Let $M$ be a real hypersurface in a non-flat complex space form $\widetilde{M}$. Then $M$ is a ruled real hypersurface if and only if $g(A X, Y)=0$ for any tangent vectors $X, Y$ of $M$ with $X, Y \perp \xi$. 
The shape operator of ruled real hypersurfaces in $P_{n} \mathbb{C}$ or $H_{n} \mathbb{C}$ is written as follows:

$$
\begin{aligned}
& A \xi=\alpha \xi+\nu W(\nu \neq 0), \\
& A W=\nu \xi, \\
& A Z=0
\end{aligned}
$$

for any $Z \perp\{\xi, W\}$, where $W \perp \xi$ is a unit vector field, $\alpha$ and $\nu$ are functions on $M$.

We may consider a question: Could we characterize a ruled real hypersurface in a non-flat complex space form in terms of the almost contact metric structure?

D. E. Blair [5] introduced a Killing tensor of type $(1,1)$. For a Riemannian manifold with Riemannian connection $\nabla$, a $(1,1)$-tensor field $T$ is said to be a Killing tensor field if it satisfies $\left(\nabla_{X} T\right) X=0$ or $\left(\nabla_{X} T\right) Y+\left(\nabla_{Y} T\right) X=0$ for any vector fields $X$ and $Y$. Then we prove:

Proposition 11. There is no real hypersurface in a non-flat complex space form whose structure tensor field $\phi$ is a Killing tensor field.

Proof. Let $M$ be a real hypersurface of $\widetilde{M}_{n}(c)$ with $c \neq 0$. Suppose that $\phi$ is a Killing tensor field. Then we get from (4)

$$
\eta(Y) A X+\eta(X) A Y-2 g(A X, Y) \xi=0 .
$$

From (16), we easily find that $A \xi=\alpha \xi$ and $A X=0$ for any vector field $X$ orthogonal to $\xi$. This says that the rank of $A$ is 0 or 1 everywhere on $M$. But, this is impossible (cf. Lemma 2.3 in [17]). This completes the proof.

Proposition 12. There is no real hypersurface in a non-flat complex space form whose shape operator $A$ is a Killing tensor field.

Proof. Suppose that $A$ is a Killing tensor field, that is, $M$ satisfies $\left(\nabla_{X} A\right) X=0$ or $\left(\nabla_{X} A\right) Y+\left(\nabla_{Y} A\right) X=0$ for any vector fields $X, Y$ on $M$. Then we have from (7)

$$
\left(\nabla_{X} A\right) Y=\frac{c}{8}(\eta(X) \phi Y-\eta(Y) \phi X)-\frac{c}{4} g(\phi X, Y) \xi
$$

for any vector fields $X, Y$ on $M$. Since $g\left(\left(\nabla_{X} A\right) Y, Z\right)=g\left(\left(\nabla_{X} A\right) Z, Y\right)$, we have from (17)

$$
c(2 \eta(X) g(\phi Y, Z)-\eta(Z) g(\phi X, Y)+\eta(Y) g(\phi X, Z))=0
$$

for any vector fields $X, Y$ on $M$. Put $X=\xi$ in (18) to get $c g(\phi Y, Z)=0$, which is impossible. This completes the proof.

For an almost contact metric manifold $(M, \eta, \phi, \xi, g)$, we call a $(1,1)$-tensor field $T$ on $M$ a transversal Killing tensor field if it satisfies $\left(\nabla_{X} T\right) X=0$ or $\left(\nabla_{X} T\right) Y+\left(\nabla_{Y} T\right) X=0$ for any vector fields $X, Y$ orthogonal to $\xi$. By (4) and Proposition 10 then we have: 
Theorem 13. Let $M$ be a real hypersurface of $\widetilde{M}_{n}(c)$ with $c \neq 0$. Then $\phi$ is transversally Killing if and only if $M$ is locally congruent to a ruled real hypersurface.

In order to determine real hypersurfaces of a non-flat complex space form with transversal Killing shape operator, we prepare the following results:

Theorem 14 ([18]). Let $M$ be a real hypersurface in a non-flat complex space form $\widetilde{M}_{n}(c), n \geq 3$. Then the shape operator $A$ is $\eta$-parallel, that is $g\left(\left(\nabla_{X} A\right) Y\right.$, $Z)=0$ for $X, Y, Z \perp \xi$ if and only if $M$ is locally congruent to a ruled real hypersurface or a real hypersurface of type $(\mathrm{A})$ or $(\mathrm{B})$ in $P_{n} \mathbb{C}$ or $H_{n} \mathbb{C}$.

Theorem 15 ([8]). Let $M$ be a real hypersurface in a non-flat complex space form $\widetilde{M}=\widetilde{M}_{n}(c)$. Then $M$ is locally congruent to a real hypersurface of type (A) or $(B)$ in $P_{n} \mathbb{C}$ or $H_{n} \mathbb{C}$ if and only if $M$ satisfies

$$
\left(\nabla_{X} A\right) Y=\eta(X)\left(\frac{c}{4} \phi Y+F Y\right)+\eta(Y) F X+g(F X, Y) \xi
$$

for any vector fields $X, Y$ tangent to $M$, where $F=\eta(A \xi) \phi A-A \phi A$.

Then, we have:

Theorem 16. Let $M$ be a real hypersurface in a non-flat complex space form $\widetilde{M}_{n}(c), n \geq 3$. Then the following conditions are mutually equivalent:

- the shape operator $A$ is transversally Killing;

- $M$ satisfies $\left(\nabla_{X} A\right) Y=-\frac{c}{4} g(\phi X, Y) \xi$ for any vector fields $X, Y$ orthogonal to $\xi$

- $M$ is locally congruent to a type (A) hypersurface.

Proof. Suppose that the shape operator $A$ is a transversal Killing tensor field. Then $M$ satisfies $\left(\nabla_{X} A\right) X=0$ or $\left(\nabla_{X} A\right) Y+\left(\nabla_{Y} A\right) X=0$ for any vector fields $X, Y$ orthogonal to $\xi$. We have from (7) that $A$ is transversally Killing if and only if

$$
\left(\nabla_{X} A\right) Y=-\frac{c}{4} g(\phi X, Y) \xi
$$

for any vector fields $X, Y$ orthogonal to $\xi$. From (20), we see at once that $A$ is $\eta$-parallel. Hence, by Theorem 14 we have that $M$ is locally congruent to a ruled real hypersurface or a real hypersurface of type $(\mathrm{A})$ or $(\mathrm{B})$ in $P_{n} \mathbb{C}$ or $H_{n} \mathbb{C}$. We first consider the case that $M$ is a ruled real hypersurface. Then, from (15) and (20) we compute

$$
\begin{aligned}
-\frac{c}{4} \xi & =\left(\nabla_{W} A\right) \phi W \\
& =\nabla_{W}(A \phi W)-A \nabla_{W}(\phi W) \\
& =-A\left(\nabla_{W} \phi\right) W-A \phi \nabla_{W} W \quad(\because(15)) \\
& =-A \phi \nabla_{W} W \quad(\because(4)) \\
& =\left(\nu-\frac{c}{4 \nu}\right) A W \quad\left(\because \nabla_{W} W=\left(\nu-\frac{c}{4 \nu}\right) \phi W(\text { cf. }[16])\right)
\end{aligned}
$$




$$
=\nu\left(\nu-\frac{c}{4 \nu}\right) \xi
$$

The equation (21) yields that $\nu=0$, which can not occur. Next, we consider the case that $M$ is a real hypersurface of type (A) or (B) in $P_{n} \mathbb{C}$ or $H_{n} \mathbb{C}$. Then using (19) and (20) we have

$$
\alpha \phi A X-A \phi A X=-\frac{c}{4} \phi X
$$

for any vector field $X$ orthogonal to $\xi$. Assume that $A X=\lambda X, X \perp \xi$. Using (9), then we get

$$
\alpha\left(\lambda^{2}-\alpha \lambda-\frac{c}{4}\right)=0
$$

where $2 \lambda \neq \alpha$. From (22) we see that $\alpha=0$ or $M$ is locally congruent to a real hypersurface of type (A) in $P_{n} \mathbb{C}$ or $H_{n} \mathbb{C}$ by using Proposition 8 . Also, we know that $\alpha \neq 0$ for a real hypersurface of type (B). As already stated in the proof of Theorem $9,2 \lambda=\alpha$ determines a horosphere in $H_{n} \mathbb{C}$. Thus we have completed the proof.

Remark 1. The above result gives an improvement of the characterization of real hypersurfaces of type (A) in a non-flat complex space form proved in [14]. Indeed, the second condition (0.4) in their theorem is redundant.

We close the present paper by raising a problem.

Problem 1. Prove Theorem 14 and Theorem 16 when $n=2$.

Acknowledgement. The author thanks to the referee for careful reading the manuscript and comments for the revised version.

\section{References}

[1] T. Adachi, M. Kameda, and S. Maeda, Real hypersurfaces which are contact in a nonflat complex space form, Hokkaido Math. J. 40 (2011), no. 2, 205-217.

[2] S.-S. Ahn, S.-B. Lee, and Y. J. Suh, On ruled real hypersurfaces in a complex space form, Tsukuba J. Math. 17 (1993), no. 2, 311-322.

[3] J. Berndt, Real hypersurfaces with constant principal curvatures in complex hyperbolic space, J. Reine Angew. Math. 395 (1989), 132-141.

[4] D. E. Blair, Riemannian Geometry of Contact and Symplectic Manifolds, Progress in Math. 203, Birkhäuser Boston, Inc., Boston, second edition, 2010.

[5] _ Almost contact manifolds with Killing structure tensors, Pacific J. Math. 39 (1971), no. 2, 285-292.

[6] T. E. Cecil and P. J. Ryan, Focal sets and real hypersurfaces in complex projective space, Trans. Amer. Math. Soc. 269 (1982), no. 2, 481-499.

[7] J. T. Cho and J. Inoguchi, Contact metric hypersurfaces in complex space forms, Proceedings of the workshop on Differential Geometry of Submanifolds and Related Topics, Saga, August 4-6, 2012.

[8] J. T. Cho and M. Kimura, Transversal symmetries on real hypersurfaces in a complex space form, Hiroshima Math. J. 43 (2013), no. 2, 223-238.

[9] G. Dileo and A. M. Pastore, Almost Kenmotsu manifolds and local symmetry, Bull. Belg. Math. Soc. Simon Stevin 14 (2007), no. 2, 343-354. 
[10] S. I. Goldberg and K. Yano, Integrability of almost cosymplectic structures, Pacific J. Math. 31 (1969), 373-381.

[11] S. Kanemaki, Quasi-Sasakian manifolds, Tôhoku Math. J. 29 (1977), no. 2, 227-233.

[12] K. Kenmotsu, A class of almost contact Riemannian manifolds, Tôhoku Math. J. 24 (1972), 93-103.

[13] U.-H. Ki and Y. J. Suh, On real hypersurfaces of a complex space form, Math. J. Okayama Univ. 32 (1990), 207-221.

[14] - On a characterization of real hypersurfaces of type $A$ in a complex space form, Canad. Math. Bull. 37 (1994), no. 2, 238-244.

[15] M. Kimura, Real hypersurfaces and complex submanifolds in complex projective space, Trans. Amer. Math. Soc. 296 (1986), no. 1, 137-149.

[16] - Sectional curvatures of holomorphic planes on a real hypersurfaces in $P^{n}(\mathbb{C})$, Math. Ann. 276 (1987), no. 3, 487-497.

[17] M. Kon, Pseudo-Einstein real hypersurfaces in complex space forms, J. Diffential Geometry 14 (1979), no. 3, 339-354.

[18] S. H. Kon and T. H. Loo, Real hypersurfaces in a complex space form with $\eta$-parallel shape operator, Math. Z. 269 (2011), no. 1-2, 47-58.

[19] Y. Maeda, On real hypersurfaces of a complex projective space, J. Math. Soc. Japan 28 (1976), no. 3, 529-540.

[20] S. Maeda and S. Udagawa, Real hypersurfaces of a complex projective space in terms of holomorphic distribution, Tsukuba J. Math. 14 (1990), no. 1, 39-52.

[21] S. Montiel and A. Romero, On some real hypersurfaces of a complex hyperbolic space, Geom. Dedicata 20 (1986), no. 2, 245-261.

[22] R. Niebergall and P. J. Ryan, Real hypersurfaces in complex space forms, Tight and taut submanifolds (Berkeley, CA, 1994), 233-305, Math. Sci. Res. Inst. Publ., 32, Cambridge Univ. Press, Cambridge, 1997.

[23] M. Okumura, Certain almost contact hypersurfaces in Kaehlerian manifolds of constant holomorphic sectional curvature, Tohoku Math. J. (2) 16 (1964), 270-284.

[24] - On some real hypersurfaces of a complex projective space, Trans. Amer. Math. Soc. 212 (1975), 355-364.

[25] - Compact real hypersurfaces of a complex projective space, J. Differential Geom. 12 (1977), no. 4, 595-598.

[26] Z. Olszak, Curvature properties of quasi-Sasakian manifolds, Tensor (N.S.) 38 (1982), 19-28.

[27] Y. J. Suh, On real hypersurfaces of a complex space form with $\eta$-parallel Ricci tensor, Tsukuba J. Math. 14 (1990), no. 1, 27-37.

[28] R. Takagi, On homogeneous real hypersurfaces in a complex projective space, Osaka J. Math. 19 (1973), 495-506.

[29] — Real hypersurfaces in a complex projective space with constant principal curvatures I, II, J. Math. Soc. Japan 15 (1975), 43-53, 507-516.

Department of Mathematics

Chonnam National University

GwanguU 500-757, Korea

E-mail address: jtcho@chonnam.ac.kr 